Check for updates

Cite this: RSC Adv., 2019, 9, 10004

Received 19th September 2018

Accepted 2nd March 2019

DOI: 10.1039/c8ra07783e

rsc.li/rsc-advances

\section{Enhancing the antitumor activity of tea polyphenols encapsulated in biodegradable nanogels by macromolecular self-assembly $\uparrow$}

\begin{abstract}
Chen Liu, ${ }^{a}$ Zhong Zhang, ${ }^{\text {*ab }}$ Qingjun Kong, ${ }^{a}$ Runguang Zhang ${ }^{a}$ and Xingbin Yang (D) *a
Nanogels (NGs) with desirable stability have emerged as a promising platform for biomedical applications. Herein, a convenient approach was developed to encapsulate and protect tea polyphenols (TPs) by macromolecular self-assembly of lysozyme (Ly) and carboxymethyl cellulose (CMC) through a heating treatment. Biodegradable Ly-CMC NGs were formed on the basis of molecules driven by electrostatic interaction and hydrophobic forces. The particle size and morphology of the Ly-CMC NGs were analyzed using a Malvern particle size analyzer, fluorescence spectrophotometer, and scanning electron microscope. The results showed that the heated NGs were spherical with better stability and smaller particle size. The encapsulation efficiency of TP-loaded NGs was $89.05 \pm 3.14 \%$, and it indicated that the Ly-CMC NGs may have a strong binding force with TPs. Moreover, TP-loaded NGs showed a sustained release feature. The DPPH and ABTS-scavenging rates of the TP-loaded NGs were $76.5 \%$ and $86.1 \%$, respectively. The antitumor activity of the TP-loaded NGs can effectively inhibit the proliferation of $\mathrm{HepG}_{2}$ cells. Furthermore, TP-loaded NGs were proven to significantly enhance the induction of apoptosis in hepatoma cells and exhibit obvious cell cycle arrest. Our results demonstrate that the LyCMC NGs have extensive application prospects as a biocompatible and biodegradable delivery carrier of food functional factors to improve their antitumor effects.
\end{abstract}

\section{Introduction}

Nanotechnology has been widely applied to form varieties of delivery systems for the encapsulation, protection and controlled release of bioactive substance and drugs; ${ }^{1}$ these delivery systems are collectively called nanosystems. According to the different assembly unit used for the macromolecule selfassembly, nanosystems can be divided into two categories: synthetic polymer systems and natural macromolecule systems., ${ }^{2,3}$ Synthetic polymer systems include but are not limited to liposomes, micelles, dendrimers, hydroxyapatite NPs and nanotubes. ${ }^{2,3}$ Many of them were dropped from further development towards reaching the clinical stage due to their insufficient in vivo biodistribution, low plasma stability, rapid plasma clearance, and high toxicity. ${ }^{4-6}$ These drawbacks may induce chronic side effects in the body and are unbeneficial to the controlled release of drugs. It was reported that several

${ }^{a}$ Shaanxi Engineering Laboratory for Food Green Processing and Safety Control, College of Food Engineering and Nutritional Science, Shaanxi Normal University, Xi'an 710119, China.E-mail: zzhang@snnu.edu.cn; xbyang@snnu.edu.cn; Fax: +8629-85310517; +86-29-85310580; Tel: +86-29-85310517; +86-29-85310580

${ }^{b}$ School of Chinese Medicine, Li Ka Shing Faculty of Medicine, The University of Hong Kong, 10 Sassoon Road, Pokfulam, Hong Kong, China

$\dagger$ Electronic supplementary information (ESI) available. See DOI: $10.1039 / \mathrm{c} 8 \mathrm{ra} 07783 \mathrm{e}$ intracellular compartments and some tumors were present in thiol-overexpressing microenvironments with more reducibility than normal extracellular spaces., ${ }^{3,7}$ Besides these natural macromolecule systems, biodegradable NGs derived from natural protein/polysaccharides have recently attracted extensive attention. Biodegradable NGs are three-dimensional hydrophilic polymer networks at nanoscale that swell in water. ${ }^{\mathbf{8 9}}$ These NGs have Hamaker constants similar to those of water and can disperse into nanosized hydrogel particles with good dispersion in aqueous solutions. ${ }^{\mathbf{1 0}}$ Compared to other nanocarriers such as dendrimers and liposomes, NGs have good biocompatibility, high aqueous dispersibility, a welldefined structure, small immunogenicity and nontoxicity. ${ }^{5}$ These advantages make them an ideal system for the loading of various biomarkers, drugs and polyphenols through proper physical or chemical conjugation. For example, polyelectrolyte complex coacervation is a convenient way to form NGs with good stability in water via electrostatic interaction. Moreover, NGs have been proven to be taken up by cells more efficiently than conventional carriers such as liposomes, which are less stable than NGs. ${ }^{5,6}$ It was also reported that, as drug carriers, NGs can significantly improve the bioavailability and in vivo safety of drugs.,10 Among them, biodegradable NGs derived from natural protein/polysaccharides are potentially controlled release carriers for drugs and polyphenols; through manipulating their physical structure or degradability under specific 
cellular conditions, they can achieve their delivery property. The final biodegraded products may result in reduced in vivo toxicity in comparison with nondegradable materials. ${ }^{11,12}$ Thus, they are excellent candidates for building bioactive polyphenol delivery systems.

Lysozyme (Ly) is a natural globular protein extracted from egg whites with positive charges and an isoelectric point of 10.7. It has a molecular weight of $14.3 \mathrm{kD}, 4$ disulfide bridges and 129 amino acid residues. ${ }^{\mathbf{1 3}}$ Like most food proteins, Ly has a good gel performance, having higher viscosity, plasticity, elasticity and water binding capacity. ${ }^{\mathbf{1 4}}$ Hence, a large number of studies use Ly and polysaccharides as a model for the construction of drug and bioactive substance delivery systems, based on its excellent intrinsic biocompatibility and biodegradability. ${ }^{\mathbf{1 3 - 1 5}}$ For example, Lin reported the effects of $\mathrm{pH}$, the protein/ polysaccharide mass ratio, ionic strength and temperature on the complex of lysozyme and pectin by measuring turbidity, zeta potential and microstructure. ${ }^{8}$ The results showed that epigallocatechin gallate (EGCG) encapsulated by the lysozyme and pectin NGs still had good antioxidant ability, up to $90 \%$ scavenging rates in DPPH and 99\% in ABTS+. Lysozyme-pectin NGs have a better embedding effect and stability than free EGCG, which has broadened the application of EGCG in food.

Carboxymethylcellulose (CMC), a typical anionic polysaccharide, is a cellulose derivative with carboxymethyl groups $\left(-\mathrm{CH}_{2}-\mathrm{COOH}\right)$ bound to some of the hydroxyl groups of the glucopyranose monomers that constitute the cellulose skeleton. ${ }^{\mathbf{1 6 , 1 7}}$ Because of its favorable biodegradability, biocompatibility and low immunogenicity, CMC has been reported as a commonly used polysaccharide in degradable NG delivery systems. ${ }^{18-20}$ For example, Liang used zein and sodium carboxymethyl cellulose to fabricate microspheres with a polymer network for the effective encapsulation and protection of Taxol. ${ }^{21}$ Besides, CMC has also been used as a reliable drug delivery system. Rokhade employed gelatin and CMC to develop microspheres with a semi-interpenetrating polymer network for the encapsulation of ketorolac tromethamine and achieved its controlled release. ${ }^{22}$ In summary, NGs based on natural proteins and polysaccharides are superior to NGs obtained from proteins alone, because polysaccharides can prevent protein aggregation, particularly at the isoelectric point of the protein. ${ }^{\mathbf{1 5}}$

Despite many advances in cancer research over the past few decades, it still remains one of the leading causes of death worldwide. ${ }^{23} \mathrm{~A}$ series of studies based on in vivo animal models and the pathologic analysis of human tumors have revealed that carcinogenesis is a multistage change process, which involves the effect of reactive oxygen species. As presented in Fig. S1A, $\dagger$ reactive oxygen species can grab electrons from human tissue cells and drive the progressive transformation of normal cells into tumor cells or cancer stem cells. The process of forming an infinite proliferation of tumor cells is irreversible. Tumor cells will gradually invade adjacent tissues to form tumors. Natural dietary tea (Camellia sinensis) and its main bioactive TPs, such as epigallocatechin gallate (EGCG), epigallocatechin (EGC), epicatechin gallate (ECG), epicatechin (EC) and theaflavin (TF), have demonstrated potential antitumor effects in different preclinical and clinical studies, ${ }^{24-27}$ and its mechanism can be explained
(Fig. S1B $\dagger$ ). ${ }^{28}$ Firstly, tumor cells are retarded at the $\mathrm{G}_{0} / \mathrm{G}_{1}$ phase and cannot enter the $S$ phase or $G_{2} / M$ phase and induce tumor cell apoptosis. Secondly, TPs have a significant inhibitory effect on DNA topoisomerase II, which promotes the unwinding of DNA strands and inhibits the binding of DNA synthesis-related enzymes to DNA. Thirdly, the biochemical activity of tumor cells is different from that of normal tissues of the original organ, and its tumor cell proliferation is rapid, the cycle is short, and it is sensitive to TPs. Normal human tissue cells have slow proliferation, long cycles, and are insensitive to TPs.

However, EGCG in TPs can quickly degrade in body fluids at pH 7.4. ${ }^{29}$ Meanwhile, it is also known that catechins are very unstable after passing through the digestion process in the salivary, gastric, and upper small intestinal phases..$^{30}$ Furthermore, TPs may be susceptible to degradation when exposed to light, heat, and oxidants, ${ }^{31}$ which is a major drawback for its clinical application. Therefore, drinking tea alone may not provide enough bioactive substances to acquire health benefits. ${ }^{32}$

Inspired by the above-mentioned studies, biodegradable NGs system fabricated from Ly and CMC with different DS values were prepared and systematically characterized. TPs were introduced to the NG complexes, and the molecular interaction between TPS and Ly-CMC NGs was investigated. To assess the potential of the TP-loaded NGs for tumor therapy, its antioxidant and antitumor activities were evaluated by DPPH and ABTS+ scavenging activities and by inhibitory effects against hepatocellular carcinoma $\mathrm{HepG}_{2}$ cells. Consequently, the TP-loaded NGs were successfully applied to the drug delivery of bioactive TPs in the cellular experiment with satisfactory antitumor activity, indicating that the Ly-CMC NGs had extensive application prospects as a biocompatible and biodegradable delivery carrier of food functional factors to improve their antitumor activity.

\section{Materials and methods}

\subsection{Materials and reagents}

Lysozyme (Ly, $M_{\mathrm{w}}=14.3 \mathrm{kDa}$ ) from chicken egg whites was purchased from MP Biomedicals, (Xi'an, China). Sodium carboxymethyl cellulose (CMC, $M_{\mathrm{w}}=250 \mathrm{kDa}$ ) with the degrees of substitution (DS) of 0.7, 0.9 and 1.2 was purchased from Acros Organics (New Jersey, USA). AO/EB, 4',6-diamidino-2phenylindole (DAPI), 3-(4,5-dimethyl-2-thiazolyl)-2,5-diphenyl2- $H$-tetrazolium bromide (MTT), and phosphate-buffered saline (PBS) were purchased from Heart Biological Technology CO., Ltd. (Xi'an, China). RPMI-1640 basal medium and fetal bovine serum (FBS) were purchased from HyClone (USA). The annexin V-FITC/PI apoptosis detection kit was obtained from Shanghai BestBio Institute (Shanghai, China). Other chemicals were of analytical grade and used without further purification. Aqueous solutions were prepared with ultrapure water (18.2 M $\Omega$ specific resistance), which was obtained from the Pall Cascada laboratory water system (Millipore, Bedford, MA, USA).

\subsection{Synthesis of Ly-CMC NGs and TP loading}

Ly and CMC were added to purified water at the same concentration of $1.0 \mathrm{mg} \mathrm{mL}^{-1}$ and gently magnetically stirred for $4 \mathrm{~h}$ at 
$25{ }^{\circ} \mathrm{C}$, respectively. The above solutions were then mixed together at a Ly: CMC weight ratio of $5: 2$. After the mixed solution was magnetically stirred for $30 \mathrm{~min}$, the resulting mixture was heated to $80{ }^{\circ} \mathrm{C}$ in the digital circulating water bath for $30 \mathrm{~min}$. As a result, the biodegradable Ly-CMC NGs were obtained, and their particle size, PDI and zeta potential at $\mathrm{pH}$ from 2 to 11 were measured.

TPs were dissolved in warm water $\left(100 \mu \mathrm{g} \mathrm{mL} \mathrm{m}^{-1}\right)$, which was used as the stock solution. Then, an appropriate volume of TP stock solution was added into the previously obtained Ly-CMC NGs, and finally, the TP-loaded NGs were obtained.

For comparison, Ly-CMC coacervates (COAs) were also prepared using the same procedures and conditions, only without the process of heating instead of stirring for $1 \mathrm{~h}$. Meanwhile, the final samples including free TPs, TP-loaded Ly, TP-loaded COAs (COAs-TP) and TP-loaded NGs (NGs-TP) were obtained to determine the process conditions.

\subsection{Characterization of TP-loaded NGs}

The mean particle diameter and surface charge of the NGs were analyzed by dynamic laser scattering (DLS) on a laser light scattering instrument (Nano ZS90 Malvern, UK) at a scattering angle of $90^{\circ}$ at $25^{\circ} \mathrm{C}$. Fluorescence spectroscopy measurements were determined using a fluorescence spectrometer (RF-6000, Shimadzu, Japan), and the silt widths of excitation and emission were 5 and $5 \mathrm{~nm}$ respectively; the excitation light was set at $280 \mathrm{~nm}$, and the emission spectra were recorded from 300 to $550 \mathrm{~nm}$ for TPs at a voltage of $400 \mathrm{~V}$. The morphology of the samples was analyzed by scanning electron microscopy (SEM). The samples were prepared by dropping the solution onto the silicon wafer firstly and then spraying gold to make the sample conductive. The images were obtained with a FE-SEM S-3400N electron microscope (Hitachi Ltd., Japan) at a voltage of $80 \mathrm{kV}$.

\subsection{Encapsulation efficiency}

The TP solution was mixed and loaded with Ly-CMC NGs. The TP-loaded NGs solution was added to a $10 \mathrm{kDa}$ MWCO Amicon filter for ultrafiltration centrifugation, which was centrifuged at $4000 \mathrm{rpm}$ for $30 \mathrm{~min}$ in a refrigerated centrifuge (ZONKIA, HC3018, China) with an angle rotor. The free TPs can pass through the ultrafiltration membrane, and the TP-loaded NGs cannot be passed because of the relatively large particle size, thereby the obtained volume of free TP solution was measured by UV spectrophotometry (UV-1100, MAPADA) at $274 \mathrm{~nm}$. The percentage of entrapment efficiency (EE) and loading capacity (LC) were defined as the following formulas:

$$
\begin{aligned}
& \mathrm{EE}(\%)=\frac{\text { Total } \mathrm{TP}-\text { free } \mathrm{TP}}{\text { Total } \mathrm{TP}} \times 100 \% \\
& \mathrm{LC}(\%)=\frac{\text { Total TP }- \text { free TP }}{W_{\mathrm{n}}} \times 100 \%
\end{aligned}
$$

where $\mathrm{EE}$ is the encapsulation efficiency, LC is the loading capacity, and $W_{\mathrm{n}}$ is the weight of the Ly-CMC NGs.

\subsection{In vitro release study}

The TP-loaded NGs solution was evaluated through in vitro release experimentation, and the released amount of TPs in the phosphate buffered saline (PBS) at $\mathrm{pH} 7.4$ was determined after $0.5,1,2,4,6,8,10,12$ and $24 \mathrm{~h}$, respectively. An aliquot of these samples was put into dialysis bags (Spectr/Por, 3500 MWCO) and dispersed in $1 / 15 \mathrm{~mol} \mathrm{~L}{ }^{-1} \mathrm{PBS}$ at $37^{\circ} \mathrm{C}$ with slow magnetic stirring. At the appropriate time, $10 \mathrm{~mL}$ of solution was removed and an equal volume of fresh solution was added. The released amount of free TPs was measured by UV spectrophotometry (UV-1100, MAPADA) at $274 \mathrm{~nm}$. All experiments were processed in triplicate. The cumulative release rate $(Q)$ of TPs at each time point was determined using the following eqn (3):

$$
Q=\frac{C_{\mathrm{t}} V+C_{\mathrm{t}} V_{\mathrm{s}}}{M} \times 100 \%
$$

where $C_{\mathrm{t}}$ is the concentration of free TPs at time $t, V$ is the solution volume, $V_{\mathrm{s}}$ is the sampling volume, and $M$ is the total mass of TPs in the TP-loaded NGs solution.

\subsection{TP thermal stability in Ly-CMC NGs}

The TP-loaded NGs and the free TPs at $200 \mu \mathrm{g} \mathrm{mL} \mathrm{m}^{-1}$ in $0.1 \mathrm{M}$ phosphate buffer ( $\mathrm{pH}$ 7.4) were analyzed by HPLC on a Waters 2489 HPLC system equipped with a UV detector at $274 \mathrm{~nm}$. TPs and encapsulated TPs were dissolved in phosphate buffer before and after heating at $80{ }^{\circ} \mathrm{C}$ for $20 \mathrm{~min}$ to assess its stability. TPs from TP-loaded NGs were extracted by liquidliquid extraction with ethyl acetate. $\mathrm{A} \mathrm{C}_{18}$ column $(4.6 \mathrm{~mm}$ i.d. $\times$ $150 \mathrm{~mm}, 5 \mu \mathrm{m}$, Agilent) was used as an analytical column. The mobile phase A was methanol; B solution was ultrapure water containing $0.1 \%$ acetic acid. A gradient program was used as follows: 0-5 min for 8\% A, 5-15 min for 8-16\% A, 15-40 min for 16-30\% A, 40-55 min for 30-60\% A, 55-60 min for $60 \% \mathrm{~A}, 60-$ $70 \mathrm{~min}$ for $60-30 \% \mathrm{~A}$, and $70-77 \mathrm{~min}$ for $30-8 \% \mathrm{~A}$. Flow rate: 1.0 $\mathrm{mL} \mathrm{min}^{-1}$; injection volume: $20 \mu \mathrm{L}$; room temperature.

\subsection{In vitro antioxidant activity}

The radical-scavenging activity of TPs was evaluated by the DPPH and ABTS+ method. ${ }^{33,34}$ In brief, a radical-scavenging activity assay was conducted as follows. The absorbance values of the samples in the presence and absence of the remnant TP, containing DPPH at $517 \mathrm{~nm}$ and ABTS+ at $734 \mathrm{~nm}$, were determined with a UV-vis spectrophotometer at room temperature, respectively. TP is a kind of powerful antioxidant and free radical scavengers. The antioxidant property of TPs is mainly based on the transfer of electrons to DPPH. The antioxidant activity of TPs was evaluated by a decrease in the percentage of DPPH and ABTS+ compared to control conditions after $30 \mathrm{~min}$ of storage in the dark. The DPPH or ABTS+ radicalscavenging activity was determined by the eqn (4).

$$
R(\%)=\left(1-A_{\mathrm{E}} / A_{\mathrm{B}}\right) \times 100 \%
$$

where $R$ is the radical-scavenging activity, and $A_{\mathrm{E}}$ and $A_{\mathrm{B}}$ are the absorbance values of the sample and the blank, respectively. 


\subsection{Cell cultures and treatments}

The human hepatoblastoma cancer cell line $\mathrm{HepG}_{2}$ was received from the cell bank of the Institute of Biochemistry and Cell Biology. The cells were cultured in RPMI-1640 basal medium supplemented by $15 \%$ fetal calf serum (FCS) and $10^{6} \mathrm{IU}$ per $\mathrm{mL}$ penicillin streptomycin. These cells were cultured in $75 \mathrm{~cm}^{2}$ flasks and trypsinized with trypsin-EDTA when the cells reached approximately $80 \%$ confluence.

\subsection{Cell viability assay}

The MTT assay is firstly described by Mossmann. ${ }^{35}$ It is used to measure cell metabolic activity, cytotoxicity, proliferation and viability. Cell viability was determined by measuring the amount of tetrazolium salt in the viable cells of each sample using untreated cells as controls.

Briefly, $\mathrm{HepG}_{2}$ cells were put into 96-well cell culture plates at a density of $1 \times 10^{5}$ cells per well in complete culture medium $(100 \mu \mathrm{L})$. The cells were incubated for $24 \mathrm{~h}$ under $5 \% \mathrm{CO}_{2}$ at $37{ }^{\circ} \mathrm{C}$. The medium was substituted with the medium containing NGs, TP-loaded NGs and free TPs at concentrations of 20, $40,60,80$, and $100 \mu \mathrm{g} \mathrm{mL}{ }^{-1}$. The uncompleted medium without serum was used as the control. Then, the cells were cultured in a humidified $5 \% \mathrm{CO}_{2}$ balanced-air incubator for 12,24 and $48 \mathrm{~h}$, respectively. After cultivation, $10 \mu \mathrm{L}$ of MTT solution $(5 \mathrm{mg}$ $\mathrm{mL}^{-1}$ ) was put into each well and the plates were incubated for another $4 \mathrm{~h}$; then, formazan solution $(100 \mu \mathrm{L})$ was added into each well of the 96-well plate to be sequentially incubated for 5$10 \mathrm{~min}$ at $37^{\circ} \mathrm{C}$, and tetrazolium dye was dissolved with vigorously stirring. The absorbance value of each well was determined by a microplate reader (BioTek Instruments, Inc. USA) at a test wavelength of $570 \mathrm{~nm}$ with $690 \mathrm{~nm}$ set as the reference wavelength. The cell viability was calculated by the following eqn (5):

$$
V(\%)=\left[\left(\mathrm{OD}_{\mathrm{eg}}-\mathrm{OD}_{690}\right) / \mathrm{OD}_{\mathrm{c}}\right] \times 100 \%
$$

where $V$ is the cell viability, $\mathrm{OD}_{\text {eg }}$ is the $\mathrm{OD}$ experimental group, and $\mathrm{OD}_{\mathrm{c}}$ is the $\mathrm{OD}$ control group.

\subsection{Antitumor activity and cell apoptosis}

The antitumor activity of TP-loaded NGs-induced apoptosis in $\mathrm{HepG}_{2}$ cells was measured using $4^{\prime}$,6-diamidino-2-phenylindole (DAPI) and acridine orange/ethidium bromide (AO/EB) double staining by a fluorescence microscope; the standard procedure was listed as followed. In brief, $\mathrm{HepG}_{2}$ cells $\left(1 \times 10^{5}\right.$ cells per $\mathrm{mL}$ ) were put into 6-well cell culture plates in $2 \mathrm{~mL}$, and the cells were incubated until reaching about $60 \%$ confluence at $37{ }^{\circ} \mathrm{C}$ in a $5 \% \mathrm{CO}_{2}$ incubator. They were then treated with TP-loaded NGs at $20,40,60,80$ and $100 \mu \mathrm{g} \mathrm{mL}^{-1}$, and the uncompleted medium (without serum) were used as the control. After $12 \mathrm{~h}$ cultivation, the cells were washed 3 times with $0.1 \mathrm{M}$ PBS, fixed with paraformaldehyde and then stained with DAPI and AO/EB (AO : $\mathrm{EB}=1: 1$ ), respectively. $10 \mathrm{~min}$ later, the cells were washed again with PBS solution and were observed and photographed using an inverted fluorescence microscope (Leica Microsystems, Ltd. Germany).
Cell apoptosis assay was detected as per the reported method with some modifications. ${ }^{36}$ In brief, $\mathrm{HepG}_{2}$ cells $\left(2 \times 10^{5}\right.$ cells per $\mathrm{mL}$ ) were placed into 6-well cell culture plates and incubated for $24 \mathrm{~h}$. The cells were treated with free TPs $\left(10 \mu \mathrm{g} \mathrm{mL}{ }^{-1}\right)$, blank NGs and TP-loaded NGs (equivalent TP concentration) for $24 \mathrm{~h}$. Then, the cells were extracted and washed with PBS twice and dispersed in $400 \mu \mathrm{L}$ of binding buffer with $10 \mu \mathrm{L}$ of PI and 5 $\mu \mathrm{L}$ of annexin V-FITC. Finally, the samples were incubated in a dark place for $10 \mathrm{~min}$ at $2-8{ }^{\circ} \mathrm{C}$ and analyzed on the flow cytometer; the excitation light was set at $488 \mathrm{~nm}$ and the emission light was recorded at $525 \mathrm{~nm}$. The number of PIpositive and annexin V-FITC-positive cells in each field of view was measured by counting the cells directly.

\subsection{Statistical analysis}

All measurements were conducted at least three times, and the results were reported as means $\pm \mathrm{SD}$ (standard deviation). The data were analyzed by a one-way analysis of variance (ANOVA) and Duncan's multiple range tests. For all analyses, $P$-values of 0.05 or less were considered to be statistically significant.

\section{Results and discussion}

\subsection{Fabrication of Ly-CMC NGs}

The Ly-CMC NGs were prepared by electrostatic interaction, macromolecular self-assembly approach. Fig. 1A displayed the three-step approaches for the preparation of Ly-CMC NGs, containing the self-assembly of lysozyme, the formation of LyCMC coacervates, and the construction of Ly-CMC NGs. ${ }^{37,38}$ Firstly, Ly nanoparticles were formed through electrostatic interaction self-assembly. Furthermore, Ly-CMC coacervates were prepared by adding Ly nanoparticles dropwise to CMC solution with slow stirring. CMC was used as the cellulose skeleton for the formation of 3D interconnected microspheres structures. The size of the Ly-CMC coacervates can be changed in the range from 420 to $720 \mathrm{~nm}$ by changing the preparation conditions such as the degree of substitution (DS), the percentage of CMC, the reaction temperature and pH. Finally, the Ly-CMC coacervates can be converted to Ly-CMC NGs through heating treatment; the particle size can reduce to $260 \mathrm{~nm}$ and provide a more stable structure to embed TPs. Fig. 1B displayed the controlled release and absorption process of TP-loaded NGs in vivo. As the surroundings pH changes, the Ly-CMC NGs become a compact compound under a gastric acidic environment and become a loose structure under alkaline conditions, so that the active substance can be controllably released in the small intestine. ${ }^{39}$ NGs act as a carrier that can enhance the efficacy of TPs; as such, NGs controlled their release content in close proximity to the target cells, attach to the membrane of the cell and act as an extracellular sustainedrelease drug depot. Small-sized NGs not only enable themselves to pass through the smallest capillaries, but also promote passive tumor targeting due to the enhanced permeability and retention (EPR) effect of the tumor vasculature. In addition, NGs can achieve active cellular targeting by functionalizing the 


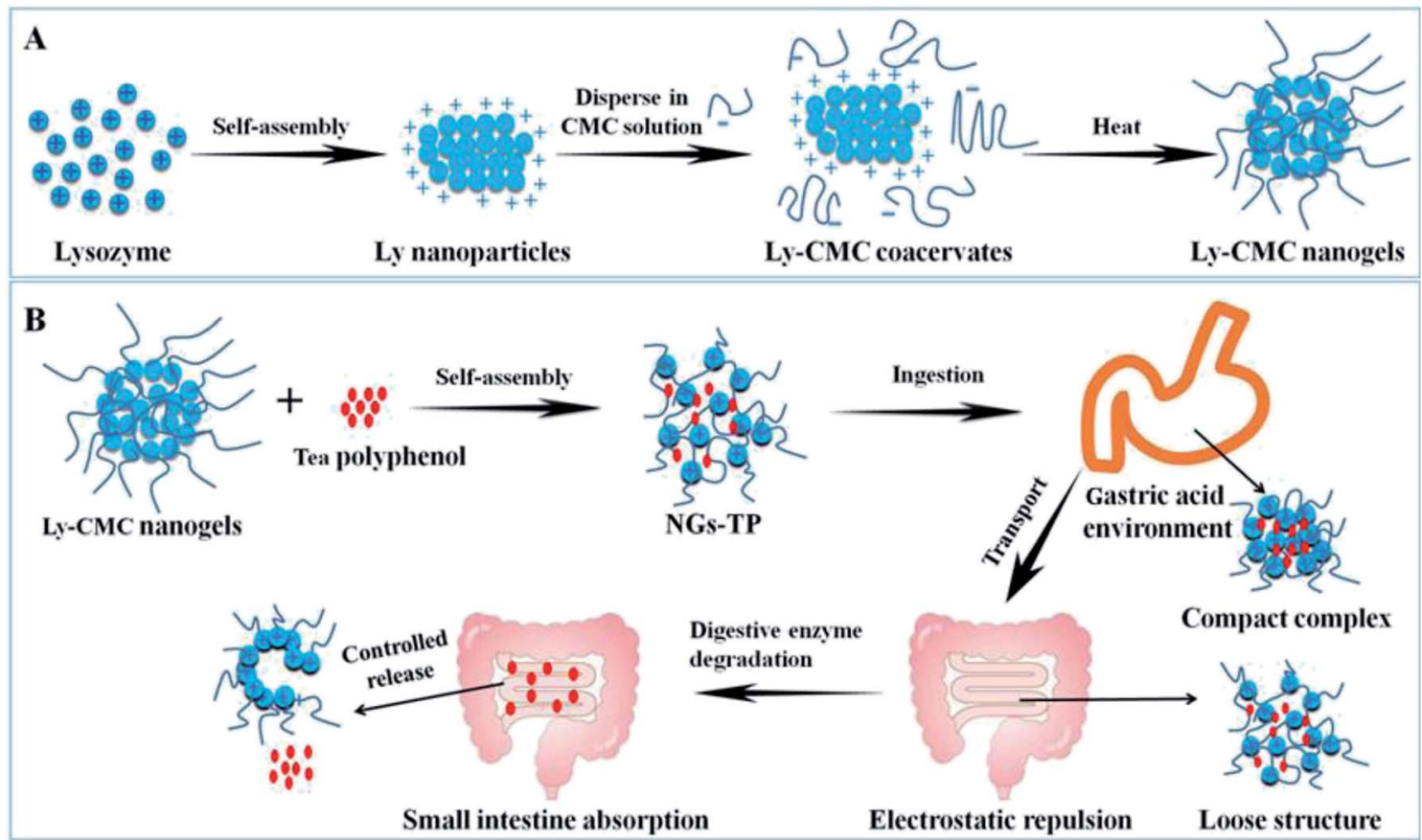

Fig. 1 (A) The three-step approach for the preparation of Ly-CMC NGs. (B) The controlled release and absorption process of TP-loaded NGs in vivo.

surface of it with ligands that promote cell-specific recognition and binding.

\subsection{Optimization of the synthesis conditions}

To achieve optimal conditions for constructing the desired LyCMC NGs, the major influence factors of Ly-CMC NGs including different $\mathrm{pH}, \mathrm{CMC}$ with different degrees of substitution, $\mathrm{NaCl}$ concentration and the ratio of Ly and CMC were tested. Besides considering the encapsulation efficiency of TPs, the loading capacity and particle size of Ly-CMC NGs should also be taken into account.

Among the chemical interactions, electrostatic interaction is a primary interaction force between proteins and polysaccharides to form a gel. The electrostatic interaction force between protein and polysaccharide composites is closely related to the charge of the biomacromolecules, and the charge of biomacromolecules is related to the solution $\mathrm{pH} .^{40}$ To explain the electrostatic interaction between Ly and CMC, the zeta potentials of both the lysozyme and the CMC solution were determined in at a pH range of 2.0-11.0 (Fig. 2A). Lysozyme solution was positively charged when the solution $\mathrm{pH}$ was lower than 9.4, which is lower than its isoelectric point of 10.7 because of the charged patches on the protein molecule surface. CMC solution was negatively charged in the wide range of $\mathrm{pH} 2.0-$ 11.0. When the mixture solution is neutral, lysozyme and CMC have positive and negative charges, respectively. Therefore, the electrostatic interaction is the main driving force between Ly and CMC.

In addition, the electrostatic attraction between positively charged Ly and negatively charged CMC could also be affected by the mass ratio of Ly and CMC. The results (Fig. 2B) showed that the mass ratios can change the particle size of Ly-CMC NGs. The average particle size of NGs was first reduced and then increased, reaching a minimum at a mass ratio of $5: 2$, which was determined by the change in the net charge of the Ly-CMC coacervates. When the mass ratio of Ly-CMC was $5: 2$, most of the lysozyme particles would bind to the CMC chain. The formed electrostatic composite had a high net negative charge such that the electrostatic composite could be kept relatively stable by electrostatic repulsion, and the particle size of the formed NGs remained relatively small. Moreover, the particle size of the Ly-CMC coacervates also increased as the DS increased. This was because the CMC with a high DS value contained more carboxyl groups, producing more negative charges on the surface of the molecule, which can bind more lysozyme to increase the particle size. According to the minimum particle size of NGs, it was demonstrated that the mass ratio of CMC is $5: 2$, and a DS of 0.7 is the optimal choice for the weight ratio. In order to compare the effects of heating, Ly-CMC coacervates (COAs) were also prepared without a heating process; the results revealed that the heated Ly-CMC NGs had better stability and a smaller particle size, because the protein can be sufficiently denatured under heating to form an intermolecular hydrophobic interaction and a disulfide bond, thereby maintaining the particle size of the assembly constant. Therefore, the NGs also used a heating process.

The influence of different $\mathrm{pH}(2.0-10.0)$ on the particle size and polydispersity index (PDI) of Ly-CMC NGs with a weight ratio of $5: 2$ during the synthesis process was shown. Because the $\mathrm{pH}$ of normal human tissues and blood is 7.4, considering the formulation of subsequent experimental conditions, $\mathrm{pH} 7.4$ was chosen as the control pH (Fig. 2C). It is well known that the 
A

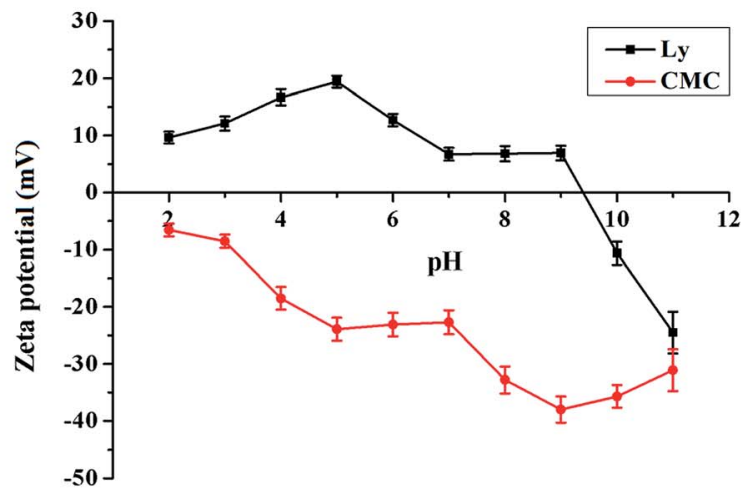

C

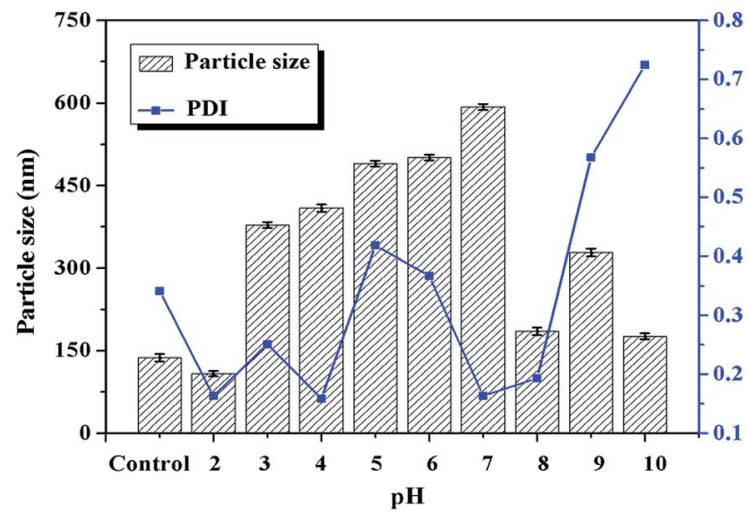

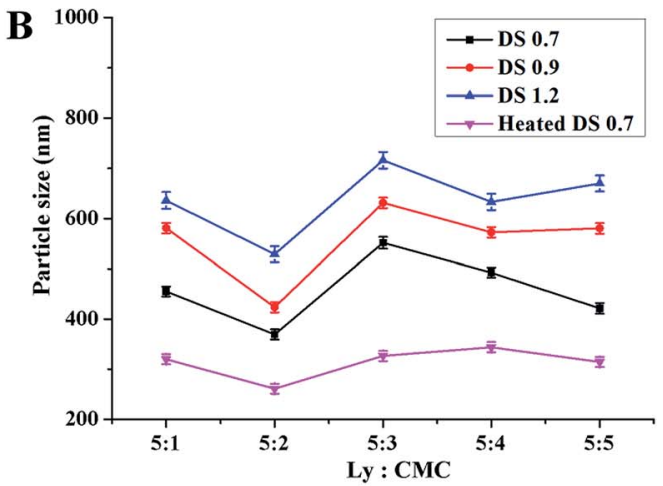

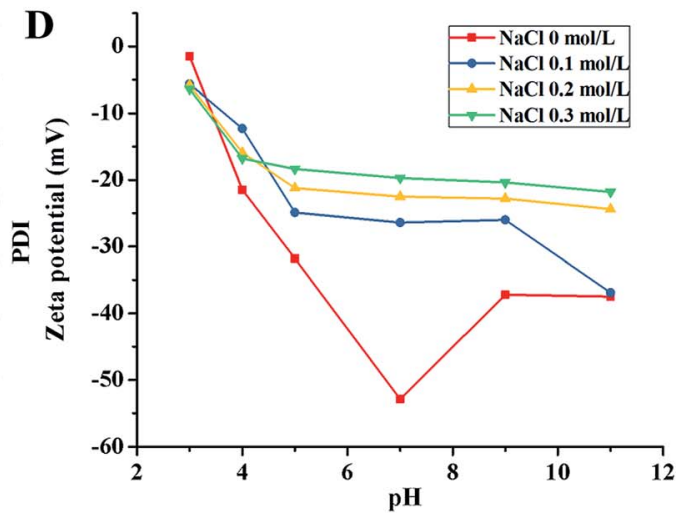

Fig. 2 (A) The zeta potential of both the lysozyme and the CMC solution in the $\mathrm{pH}$ range of 2.0-11.0. (B) The effect of the weight ratio of Ly and CMC and the degree of substitution of CMC on the particle size of Ly-CMC NGs. (C) The effect of pH on the particle size and PDI of Ly-CMC NGs from 2.0 to 10.0 during the synthesis process; control pH was set at $\mathrm{pH} 7.4$. (D) The effect of different $\mathrm{NaCl}$ concentrations on the zeta potentials of NGs in the $\mathrm{pH}$ range of 2.0-11.0.

smaller the particle size and PDI, the more uniform and stable the distribution of the NGs. So it can be seen from Fig. 2C that at $\mathrm{pH} 2.0$ and 7.4, the average particle size and PDI of NGs are small and could be used in future studies. Considering that TPs were stable under acidic conditions, they are very unstable in neutral and alkaline solutions. ${ }^{29}$ The solution at $\mathrm{pH} 2.0$ was chosen as the optimum $\mathrm{pH}$ value for the synthesis of NGs in the subsequent study. This is because both Ly and CMC have opposing charges, forming a soluble electrostatic composite. When the charges of Ly and CMC are not equal and the formed composite has sufficient net charge in the outer layer, the composite is stable. If the charges of Ly and CMC are equivalent, the formed composite is not charged and the composite may be unstable and agglomerated, resulting in an increase in particle size. According to the measurements, when the $\mathrm{pH}$ is 2 and 7.4, the net charge of the complex at that $\mathrm{pH}$ is $6.2 \mathrm{mV}$ and $-50.3 \mathrm{mV}$, respectively. The Ly-CMC NGs can be stabilized with a repulsive force, which is why the Ly-CMC NGs had a smaller particle size at $\mathrm{pH} 2$ and 7.4. Due to tea polyphenols being stable under acidic conditions; they are very unstable in neutral and alkaline solutions. ${ }^{29}$ The solution at $\mathrm{pH} 2.0$ was chosen as the optimum $\mathrm{pH}$ value for the synthesis of NGs in the subsequent study. The PDI of NGs were all larger than 0.5 , because of the uneven length of the CMC chain, resulting in a non-uniform composite.

Salts can change the ionic strength of the system and regulate many types of electrostatic interactions in the solution, such as intermolecular interactions. Fig. 2D showed that the $\mathrm{NaCl}$ solution can shield the electric charge, reduce the potential of NGs, and weaken the electrostatic effect inside NGs. The results also demonstrated that the increase of $\mathrm{NaCl}$ concentration can further shield the charge, so that the electrostatic repulsion between NGs decreases, NGs aggregate, and turbidity increases, which is consistent with the reported results. ${ }^{41}$

\subsection{Characteristics of TP-loaded NGs}

The SEM images were employed to illustrate the surface morphology of Ly-CMC COA, Ly-CMC NGs and TP-loaded NGs, which were fabricated by Ly and CMC with a mass ratio of $5: 2$ and heated for $30 \mathrm{~min}$. From the SEM images (Fig. S2 $\dagger$ ), the results indicated that treatment of Ly-CMC COA for $10 \mathrm{~h}$ in the presence of chitosanase resulted in significant agglomeration of Ly-CMC COA and increased particle size (Fig. S2A and B †े). When treated with Ly-CMC NGs for $10 \mathrm{~h}$, the sample in the presence of chitosanase lost its original 3D shape (Fig. S2C and $\mathrm{D} \uparrow$ ), indicating that the Ly-CMC NGs was dissociated and agglomerated by cleavage under a reducing condition. From the SEM images (Fig. S3 $†$ ), the Ly-CMC NGs appeared as a solid microsphere with a rough surface, and the particle size is relatively uniform and partially adhered, which may be formed during the drying process. The average size of Ly-CMC NGs was much smaller than that acquired from the DLS method. This is because the DLS method can provide the radius after the NGs absorb water in solution, while SEM showed the images of the 
NGs dried on silicon wafers in a vacuum drying oven. The SEM images of NGs showed no obvious changes in morphology before or after drug loading.

The synchronous fluorescence spectrum is usually applied to measure changes in the microenvironment of amino acid residues in proteins; the polarity of the amino acid residues was determined by the shift of the fluorescence peak. Ly has six tryptophan (Trp) and three tyrosine (Tyr) residues; through the possible peak shifts of the residues, the polarity changes of the Trp and Tyr residues can be found. In regards to the fluorescence spectra of free Ly without heating at different $\mathrm{pH}$, the Ly solution has a characteristic fluorescence peak at $345 \mathrm{~nm}$. At $\mathrm{pH}$ 8 , the fluorescence intensity reaches a maximum (Fig. 3A). Concerning the fluorescence spectra of free Ly at different $\mathrm{pH}$ with heating, it can be seen that Ly is relatively stable at pH 5 after heating, but at $\mathrm{pH} 11$, the fluorescence intensity also shows a downward trend (Fig. 3B). Therefore, under acidic conditions, Ly can resist the denaturation of heating; whereas under neutral and alkaline conditions, Ly is easily degenerated, causing a decrease in fluorescence intensity. The fluorescence intensity of COAs increased as the $\mathrm{pH}$ value increased and the fluorescence peak gradually red shifted (Fig. 3C), indicating that the hydrophilicity of the microenvironment around tryptophan and tyrosine associated with Ly endogenous fluorescence was enhanced and that the complex gradually dissociated with increasing $\mathrm{pH}$. The fluorescence intensity at $\mathrm{pH} 8$ also increased in NGs, but the fluorescence intensity decreased at $\mathrm{pH} 11$ (Fig. 3D). Therefore, the solution in $\mathrm{pH} 8$ emits the strongest fluorescence, which is the optimal condition for determination.

\subsection{Encapsulation efficiency of TP loading}

The Ly-CMC NGs were prepared to overcome the shortcomings of TPs, having many biological activities but poor stability in vivo. The low stability results in low bioavailability that limits its application in the biomedical and nutritional areas.

According to the reported study, the chains of CMC can form strong hydrogen bonds with the phenolic hydroxyl group in TP molecules to create intermolecular complexes, achieving a loading effect. ${ }^{42}$ When it is heated to $80{ }^{\circ} \mathrm{C}$, the complexes change from chains to NGs, leading to a higher encapsulation efficiency. According to the measurement, the encapsulation efficiency and loading capacity of the Ly-CMC NGs for TPs were $89.05 \% \pm 3.14 \%$ and $12.71 \pm 0.44 \%$ at $\mathrm{pH} 7.4$, respectively, which indicated that approximately $90 \%$ of TPs were successfully loaded into the interior of Ly-CMC NGs. This indicated that Ly-CMC NGs may have a strong binding force with TPs.

\subsection{In vitro release study and TP protection}

The cumulative amounts of free TPs and TPs released from the Ly-CMC NGs at different pH were shown (Fig. 4). As a comparison experiment, free TP was also determined under the same
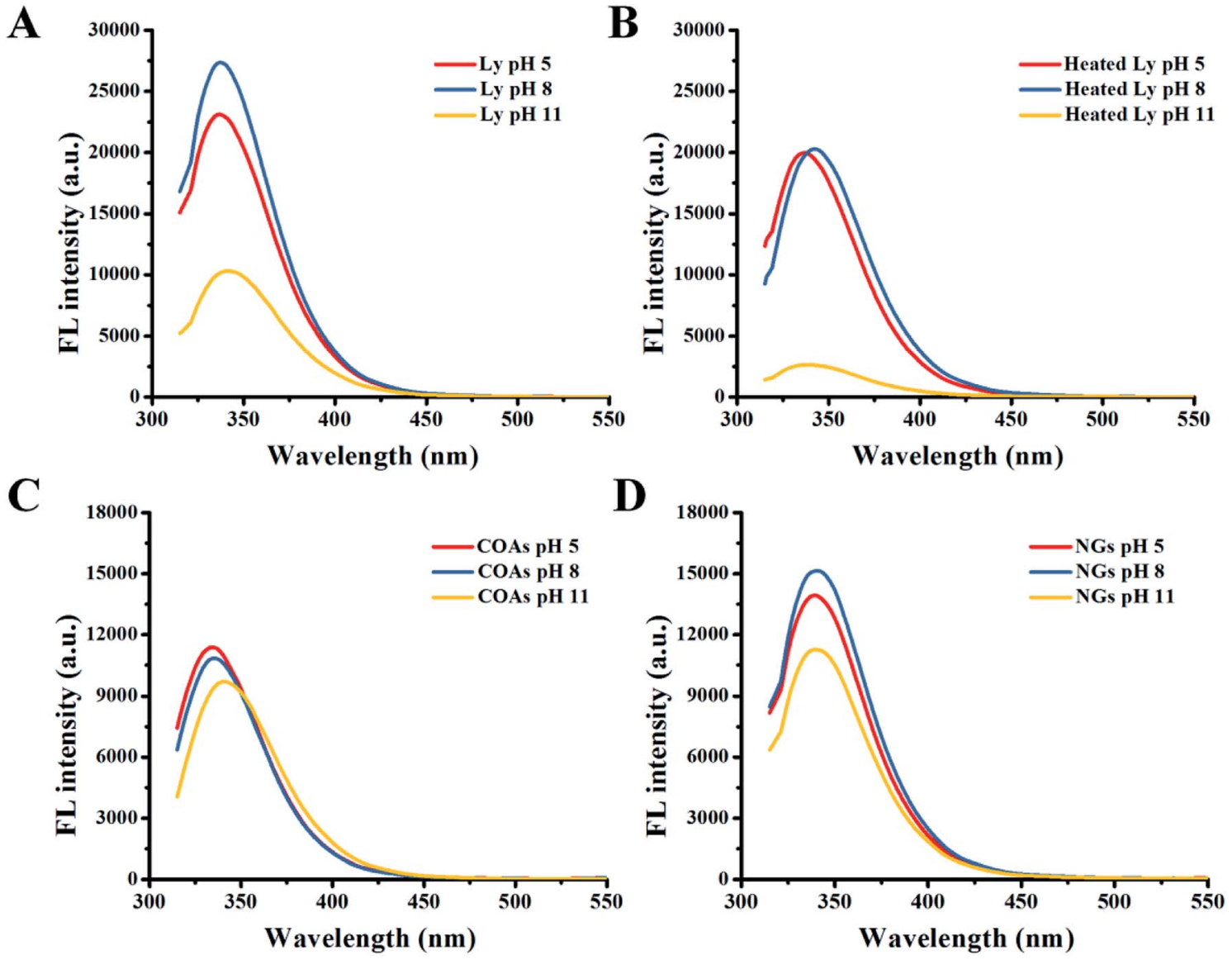

Fig. 3 The fluorescence spectrum of different solutions at pH 5, 8 and 11. (A) Free Ly. (B) Heated Ly; (C) COAs; (D) NGs. 


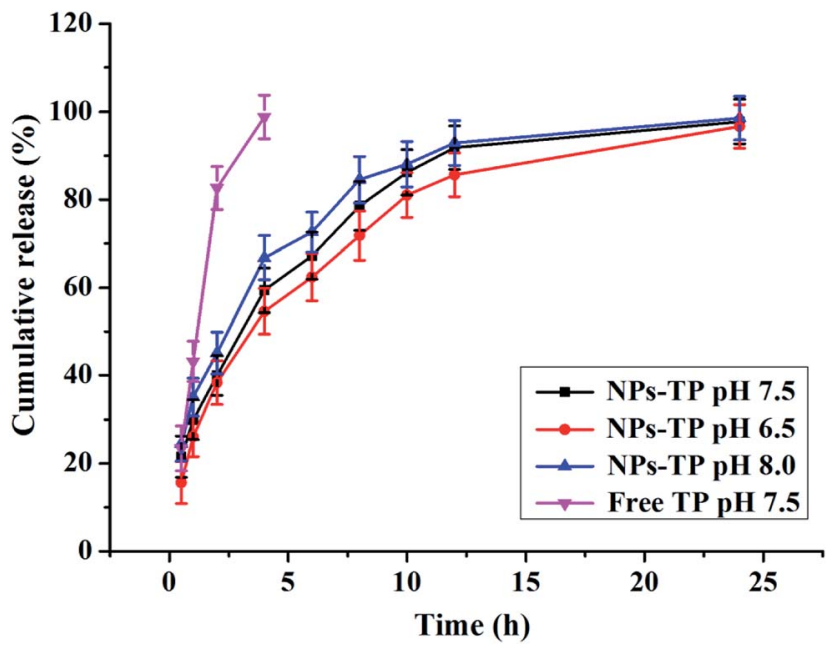

Fig. 4 The $\mathrm{pH}$-dependent cumulative amount of free TP and TP released from NGs in vitro at $\mathrm{pH} 6.5,7.5$ and 8.0.

conditions; free TPs showed a rapid release of about $83 \%$ and $99 \%$ after $2 \mathrm{~h}$ and $4 \mathrm{~h}$, respectively. The release profiles of TPs from Ly-CMC NGs at pH 6.5, 7.5 and 8 were also detected. The results possessed obvious controlled release characteristics; the release amounts reached $59.4 \%, 54.6 \%$ and $66.8 \%$ at $\mathrm{pH} 7.5,6.5$ and 8.0 after $4 \mathrm{~h}$ and $97.8 \%, 96.7 \%$ and $98.6 \%$ after $24 \mathrm{~h}$, respectively. It was found that TP release trends were similar at different $\mathrm{pH}$ conditions, and with the increase of $\mathrm{pH}$, TP release was slightly faster in alkaline $\mathrm{pH}$ than that in acidic $\mathrm{pH}$. At $12 \mathrm{~h}$, the release rates of TP-loaded NGs at pH 6.5, 7.5 and 8 were $85.62 \%, 91.84 \%$ and $92.92 \%$, respectively. At $24 \mathrm{~h}$, the release rates of TP-loaded NGs at pH 6.5, 7.5 and 8 were $96.7 \%, 97.78 \%$ and $98.58 \%$, respectively. This was because NGs were more unstable under alkaline conditions as well as the ease of releasing encapsulated TPs. ${ }^{29}$
In addition, the best-obtained release behavior of TP-loaded NGs (pH 6.5) was fitted using the zero-order and Gauss equation models. The Gauss equation model yielded the best fit, with the highest correlation coefficient $R^{2}=0.9827$ (Fig. S4A and $\mathrm{B} \dagger$ ). Therefore, the data obtained from the release studies of TPloaded NGs may follow the Gauss equation model, which assumes that the TP-loaded NGs have significant drug release characteristics.

According to the literature, ${ }^{29}$ the stability of TP is poor, and it is easy to undergo oxidation, polymerization, condensation and other reactions under conditions of humidity and sunlight. The degradation process under heating conditions may be more rapid. Furthermore, the effect of encapsulation and loading with Ly-CMC NGs on the stability of TPs was analyzed by HPLC. The results showed only $42.5 \%$ of TPs remained in phosphate buffer after 20 min of heating, and the retention time of TP peak is advanced (Fig. S5A and $\mathrm{B} \dagger$ ). This demonstrated that TPs have poor stability at high temperatures. According to the results of in vitro sustained-release experiments, the TP monomer will rapidly degrade in the phosphate buffer ( $\mathrm{pH}$ 7.4), while the NGs have the drug sustained-release ability to prolong the degradation time, and the time is up to 24 hours. In comparison, LyCMC NGs encapsulation significantly increases the stability of TPs; after incubation for $24 \mathrm{~h}$ and $48 \mathrm{~h}, 65 \%$ and $57.5 \%$ of the TP can still remain stable in the phosphate buffer $(\mathrm{pH} 7.4)$ (Fig. S5C and $\mathrm{D} \dagger$ ). These findings strongly prove that Ly-CMC NGs could effectively protect TPs against heating treatment. It was demonstrated that Ly-CMC NGs were efficient and biodegradable nanocarriers of TPs and could potentially protect and load other unstable beneficial compounds.

\subsection{Scavenging activity of TP-loaded NGs}

DPPH and ABTS+ tests have been widely applied to evaluate the scavenging ability of free radicals and antioxidant capacity. TPs
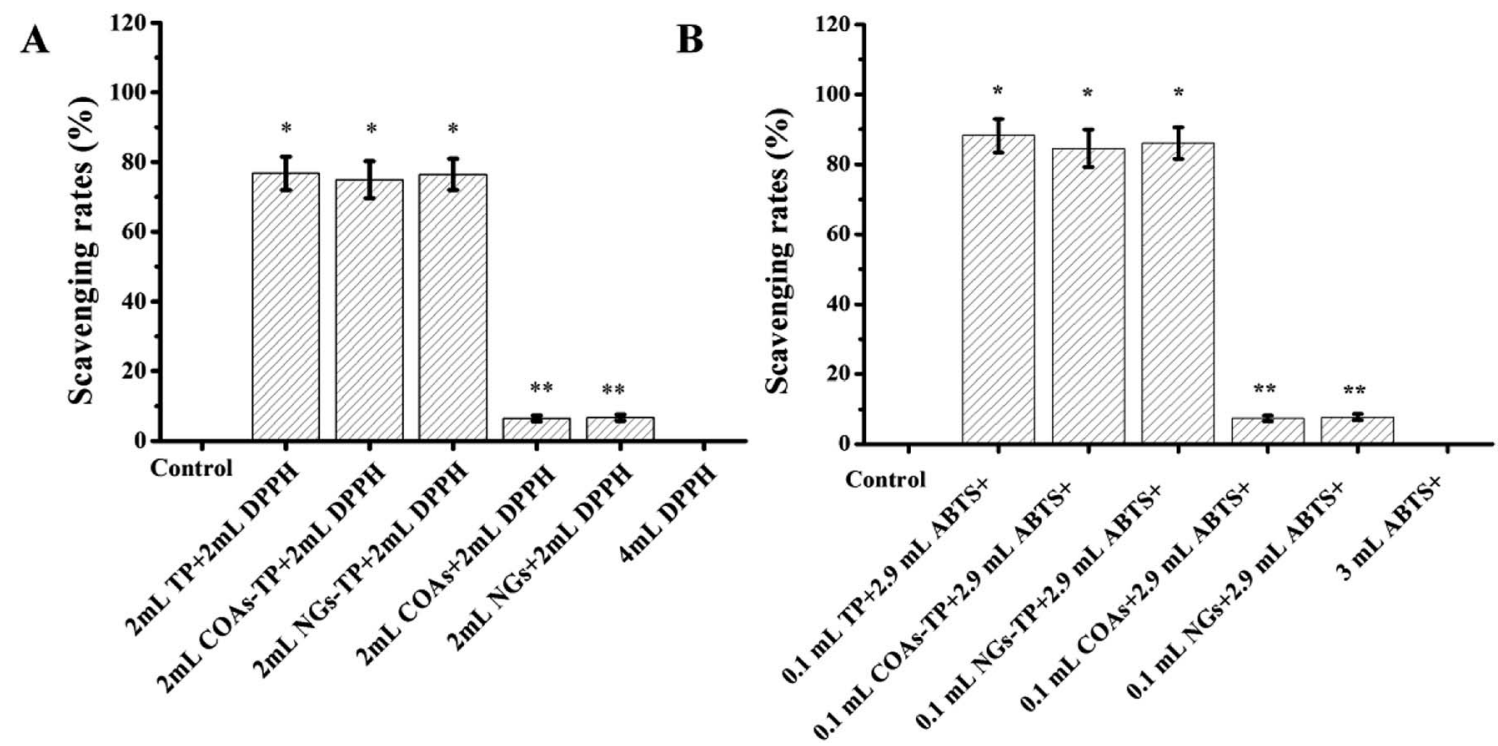

Fig. 5 The scavenging activity of TP, TP-loaded COAs and TP-loaded NGs. (A) DPPH scavenging rates. (B) ABTS+ scavenging rates. The results are expressed as the mean \pm SD of three independent experiments. $* P<0.05, * * P<0.01$ vs. the free TP group. 


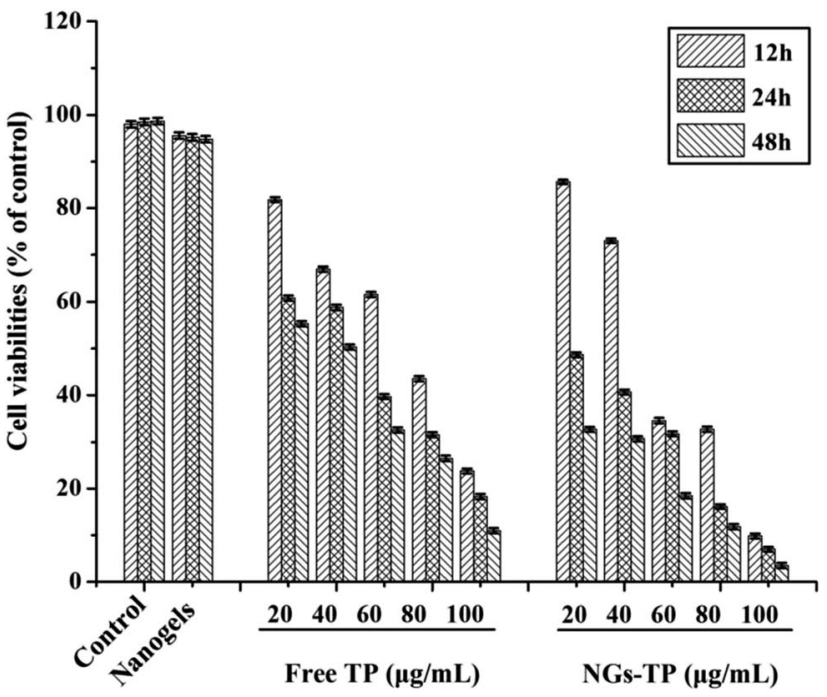

Fig. 6 Cell viabilities of TP, blank NGs and TP-loaded NGs against $\mathrm{HepG}_{2}$ cells. HepG 2 cells $\left(1 \times 10^{5}\right.$ cells per $\left.\mathrm{mL}\right)$ were incubated with different concentrations of TP and TP-loaded NGs for 12, 24 and $48 \mathrm{~h}$. All data were processed from three independent experiments $(p<$ 0.05).

have antioxidant properties, and their antioxidant strength can be used as an important indicator for investigating the biological activity of TPs. When the antioxidant is present in the solvent, the maximum absorbance at $517 \mathrm{~nm}$ and $734 \mathrm{~nm}$ for $\mathrm{DPPH}$ and ABTS+ decreases; the strength of the antioxidants is evaluated by the degree of attenuation of this absorbance value. When reacted with a free radical quencher, the color of the DPPH free radicals in the solution will change from deep violet color to colorless or pale yellow. ${ }^{43}$

The scavenging ability of the various compounds was summarized. It could be seen that CMC almost did not reduce the DPPH or ABTS+ absorption as the control, while Ly-CMC NGs and COAs could slightly reduce the absorption, which was nearly neglected compared with the scavenging rate of the TPs (Fig. 5A and B). Natural Ly was reported to possess some antioxidant activity depending on the level, sequence, and composition of the amino acids. ${ }^{\mathbf{4 4}, 45}$ The ability of TP-loaded NGs to scavenge free radicals was similar to that of free TPs, demonstrating that the antioxidant capacity of encapsulated TPs does not decrease. It was noteworthy that the TP-loaded NGs showed better radical scavenging ability than TP-loaded COAs. Because the hydrophobic group of Ly will be exposed to the outside after heating the COAs, the hydrophobic interaction of Ly and CMC is further increased to form smaller and tighter Ly-CMC NGs. Hence, the tighter structure of the Ly-CMC NGs will contribute to the encapsulation and protection of TPs.

\subsection{Cell viability}

TPs have a strong correlation with macronutrients such as proteins and polysaccharides. ${ }^{46}$ Previous results indicate that Ly-CMC NGs could load and protect TPs in an aqueous medium. To further investigate the enhancing effect of the encapsulation of TPs, the effect of antitumor activity in TPloaded NGs was investigated.

The cytotoxicity of TPs and TP-loaded NGs on $\mathrm{HepG}_{2}$ cells at concentrations ranging from 20 to $100 \mu \mathrm{g} \mathrm{mL}{ }^{-1}$ were assessed by an MTT method. After treatment with different concentrations of free TPs and TP-loaded NG samples, the growth of $\mathrm{HepG}_{2}$ cells was inhibited to varying degrees of inhibition after 12, 24 and $48 \mathrm{~h}$, representing a dose-effect relationship for different mass concentrations. The comparison of the proliferation inhibition rates of TPs and TP-loaded NGs at different concentrations was shown (Fig. 6). The results showed that with the increase of TP and TP-loaded NG concentration, the cell survival rate showed a more obvious downward trend, inversely proportional to the treatment time at the same sample concentration. The longer the treatment time, the lower the cell survival rate was. When the sample concentration was below 60 $\mu \mathrm{g} \mathrm{mL} \mathrm{m}^{-1}$ and the time is less than $24 \mathrm{~h}$, the TP-loaded NGs had
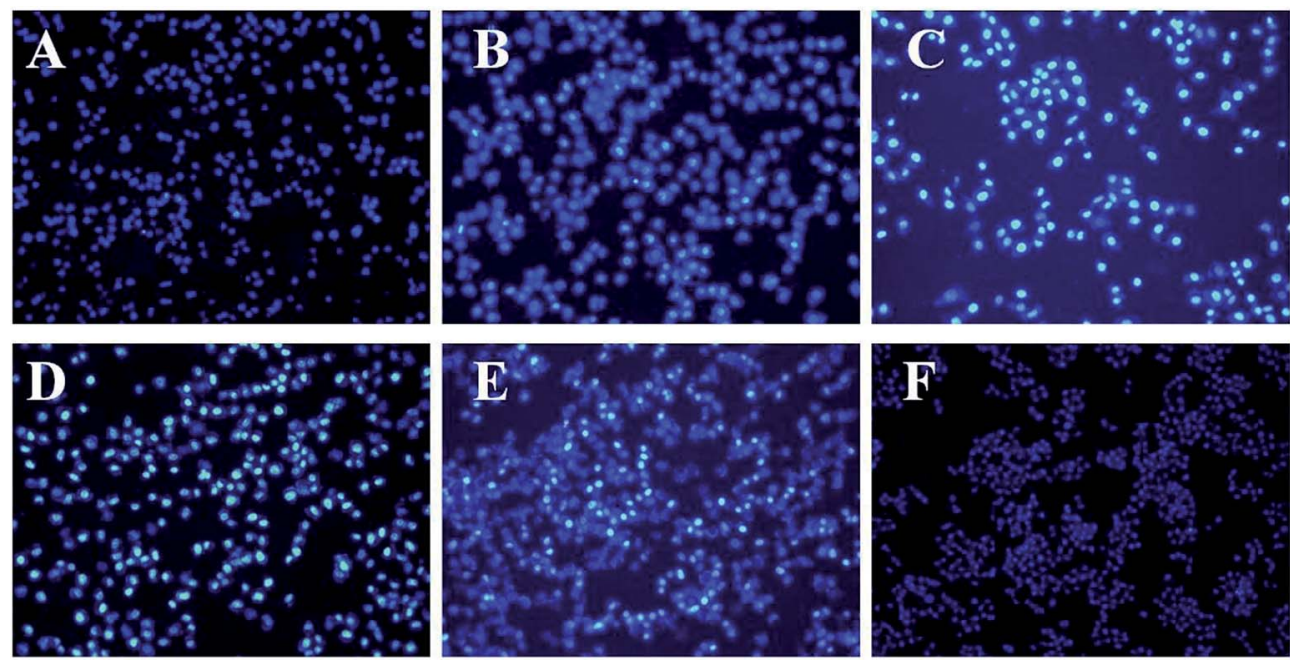

Fig. 7 The fluorescence microscope image of the $\mathrm{HepG}_{2}$ tumor cells with different TP concentrations in DAPI staining. (A-E) TP-loaded NGs at concentrations of $20,40,60,80$, and $100 \mu \mathrm{g} \mathrm{mL}^{-1}$. (F) The control group (incomplete medium). 

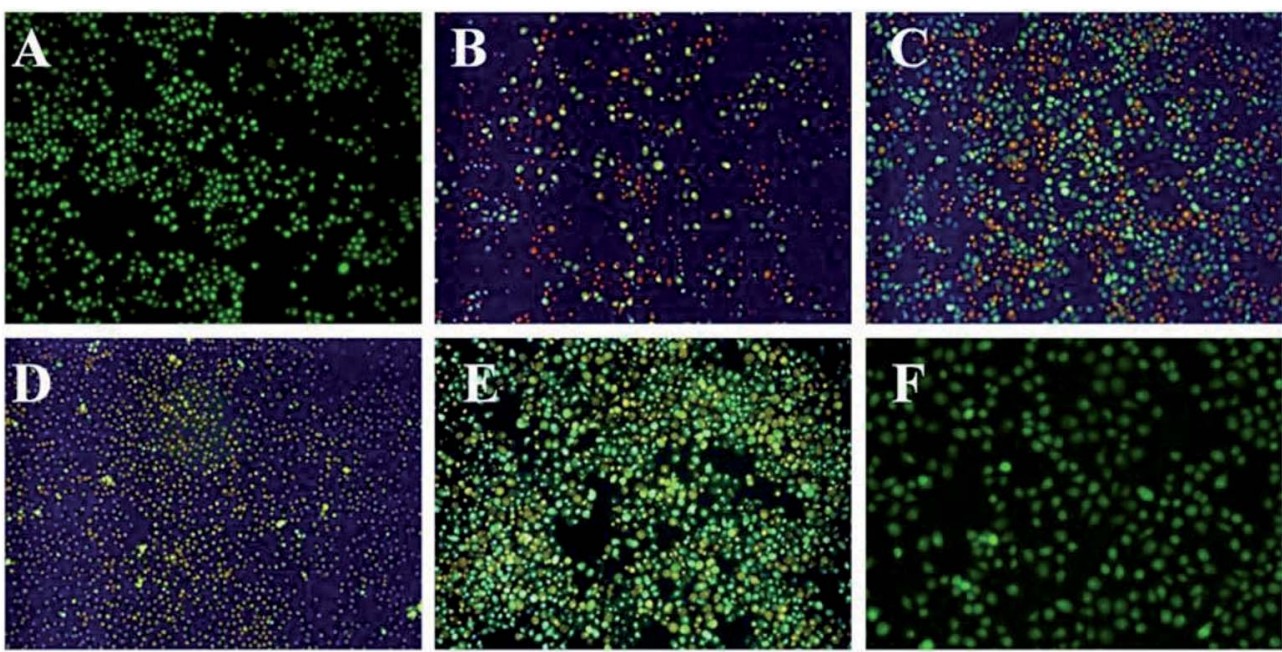

Fig. 8 The fluorescence microscope image of $\mathrm{HepG}_{2}$ tumor cells with different TP concentrations in AO/EB staining. (A-E) TP-loaded NGs at concentrations of $20,40,60,80$, and $100 \mu \mathrm{g} \mathrm{mL}^{-1}$. (F) The control group (incomplete medium).
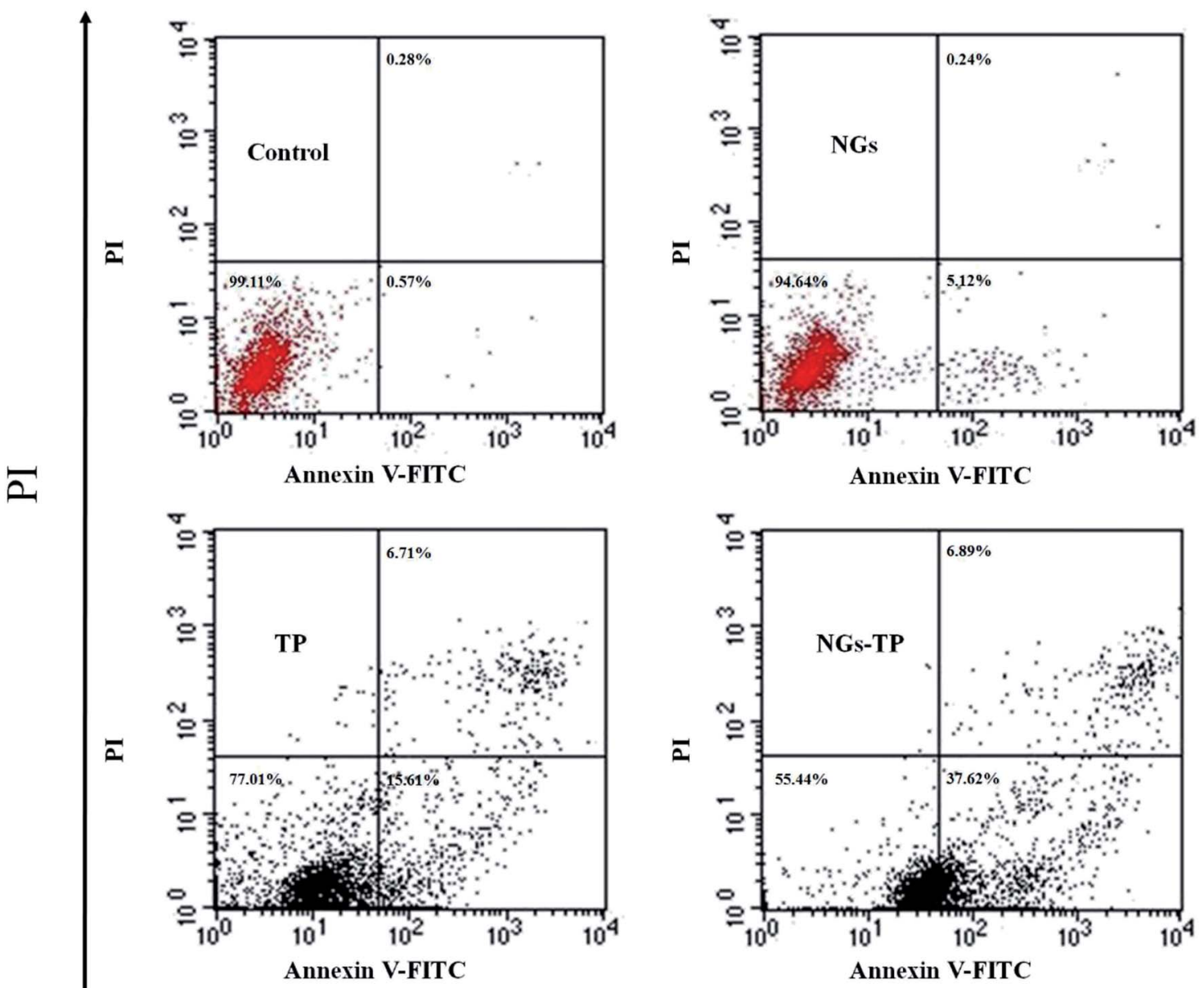

\section{Annexin V-FITC}

Fig. 9 Cell apoptosis of the HepG 2 tumor cells was performed by flow cytometry after treatment with blank NGs (control), free TP, and TPloaded NGs for $24 \mathrm{~h}$, and the TP concentration was $10 \mu \mathrm{g} \mathrm{mL}^{-1}$. 
a low inhibition rate compared to TPs. However, with the increasing concentration of TP-loaded NGs, cell viabilities also gradually decreased. Compared with $12 \mathrm{~h}$ and $24 \mathrm{~h}$ treatment groups, TP-loaded NGs in the $48 \mathrm{~h}$ treatment group could better inhibit the cell activity of cancer cells. Therefore, it can be concluded that the TP-loaded NGs displayed controlled release in vitro, but still had a significant inhibitory effect on $\mathrm{HepG}_{2}$ cells. Moreover, the Ly-CMC NGs could effectively enter into the cells through endocytosis., ${ }^{\mathbf{8} 47}$ This phenomenon revealed that the obtained TP-loaded NGs could effectively inhibit the proliferation of $\mathrm{HepG}_{2}$ cells $(p<0.01)$.

\subsection{Antitumor activity and cell apoptosis}

Based on the above MTT assays, the morphological changes of cells were further studied using DAPI staining and AO/EB double fluorescent staining. The ontological damage to cells under non-pathological conditions was noted; the active death process to better adapt to the living environment is the apoptosis of cells. The generation of apoptosis often has some representative morphological features, such as chromatin shrinkage, nuclear lysis, etc., which can be observed under a fluorescence microscope after staining.

The cell nucleus with fluorescent brightening after DAPI staining was also shown; the control group has many cells with good morphology, whereas in the sample treatment group (20 $\mu \mathrm{g} \mathrm{mL}^{-1}$ ), a small number of cells appeared round or irregular in form. Moreover, as the TP concentration increased, the cells appeared to increase the gap between the cytoplasm and the nucleus and showed nuclear fragmentation; cell death increased and the number of adherent cells also decreased. Irregular cell debris appeared in the background of the fluorescence microscope image (Fig. 7), which also indicates that the cell membrane was destroyed.

According to the AO/EB results (Fig. 8), $\mathrm{HepG}_{2}$ cells in the control group showed uniform green fluorescence under an inverted fluorescence microscope. After $12 \mathrm{~h}$ cultivation with different concentrations of TP-loaded NGs samples, the cells began to appear orange in color. As the concentration of TP increased, the proportion of orange cells increased gradually. When the TP concentration reached $100 \mu \mathrm{g} \mathrm{mL}^{-1}$, most $\mathrm{HepG}_{2}$ cells were stained orange and the morphology of the cells changed, becoming circular and irregular in shape. This is consistent with previous MTT results. These results demonstrated that the TP-loaded NGs had a stronger antitumor effect than free TPs and could be used for enhancing the activity and bioavailability of TPs.

The Annexin V-FITC/PI assay was measured by flow cytometry to evaluate the apoptosis ratio and period of the $\mathrm{HepG}_{2}$ cells. The percentage of early apoptotic cells of $\mathrm{HepG}_{2}$ treated with blank NGs was slightly higher than that of the control group, indicating that blank NGs had a slight effect on cell apoptosis. When $\mathrm{HepG}_{2}$ cells were cultured with free TPs at a concentration of $10 \mu \mathrm{g} \mathrm{mL}{ }^{-1}$, the apoptotic ratio of early cells increased significantly to $15.61 \%$, whereas it was reached $37.62 \%$ after treatment with TP-loaded NGs, in contrast with the control cells, respectively $(p<0.01, p<0.01)$. Furthermore, free
TPs could result in $6.71 \%$ of late apoptotic cells compared to untreated control cells, and TP-loaded NGs resulted in an apoptosis rate of $6.89 \%(p<0.01)$ (Fig. 9). It was important that the proportion of total apoptotic cells treated with TP-loaded NGs was remarkably higher than that in the group treated with free TPs $(p<0.01)$. The results clearly demonstrated that TP-loaded NGs had strong antitumor effects compared to free TPs. This phenomenon can be explained that TP itself is primarily responsible for its biological activity, while its degradation of product activity was reduced. Ly-CMC NGs can encapsulate TPs to make them highly stable without affecting activity, which further enhances the antitumor effects of TPs.

\section{Conclusion}

In summary, we successfully developed an effective strategy to encapsulate and protect TPs through macromolecular selfassembly interactions between the positive Ly and negative CMC. As a control, Ly-CMC COAs without heating treatment were also measured. The zeta potential results showed that electrostatic interaction was the major force for fabricating LyCMC NGs, and the protein can be denatured under heating to form an intermolecular hydrophobic interaction and a disulfide bond, thereby keeping the particle size of the NGs stable. Besides, the in vitro release experiments showed that Ly-CMC NGs had a sustained release effect and suggested that TPs were successfully encapsulated in Ly-CMC NGs. Furthermore, TPloaded NGs exhibited stronger antitumor activity on $\mathrm{HepG}_{2}$ cells than free TPs; this attractive assembly strategy allowed the reversible, biodegradable and easy modification of intelligent NGs, making it promising for diverse future applications ranging from advanced controlled release to targeted drug delivery. These results reveal the potential of Ly-CMC NGs as excellent biocompatible, biodegradable nanocarriers for the delivery of food functional factors to improve their antitumor effects.

\section{Conflicts of interest}

There are no conflicts to declare.

\section{Acknowledgements}

This work was financially supported by the National Natural Science Foundation of China (31701705, 31671823), the Hong Kong Scholars Program (XJ2017060), the Key Laboratory of Coastal Environmental Processes and Ecological Remediation, (2018KFJJ01), and the Fundamental Research Funds for the Central Universities in China (GK201803084).

\section{References}

1 I. J. Joye, G. Davidov-Pardo and D. J. McClements, Nanotechnology for increased micronutrient bioavailability, Trends Food Sci. Technol., 2014, 40, 168-182.

2 N. R. Kuznetsova, C. Sevrin, D. Lespineux, N. V. Bovin, E. L. Vodovozova, T. Mészáros, J. Szebeni and C. Grandfils, 
Hemocompatibility of liposomes loaded with lipophilic prodrugs of methotrexate and melphalan in the lipid bilayer, J. Controlled Release, 2012, 160, 394-400.

3 Y. L. Li, Y. Xiao and C. S. Liu, The horizon of materiobiology: A perspective on material-guided cell behaviors and tissue engineering, Chem. Rev., 2017, 117, 4376.

4 Y. Sun, Y. H. Huang, S. Q. Bian, J. Liang, Y. J. Fan and X. D. Zhang, Reduction-degradable PEG-b-PAA-b-PEG triblock copolymer micelles incorporated with MTX for cancer chemotherapy, Colloids Surf., B, 2013, 112, 197-203.

5 M. Gonçalves, D. Maciel, D. Capelo, S. L. Xiao, W. J. Sun, X. Y. Shi, J. Rodrigues, H. Tomaś and Y. L. Li, Dendrimerassisted formation of fluorescent nanogels for drug delivery and intracellular imaging, Biomacromolecules, 2014, 15(2), 492-499.

6 P. Ji, B. J. Zhou, Y. Zhan, Y. F. Wang, Y. H. Zhang, Y. L. Li and P. X. He, Multistimulative nanogels with enhanced thermosensitivity for intracellular therapeutic delivery, ACS Appl. Mater. Interfaces, 2017, 9(45), 39143-39151.

7 Y. Zhan, M. Gonçalves, P. P. Yi, D. Capelob, Y. H. Zhang, J. Rodrigues, C. S. Liu, H. Tomás, Y. L. Li and P. X. He, Thermo/redox/pH-triple sensitive poly $(\mathrm{N}$ isopropylacrylamide-co-acrylic acid) nanogels for anticancer drug delivery, J. Mater. Chem. B., 2015, 3, 42214230 .

8 L. F. Lin, W. Xu, H. S. Liang, L. He, S. L. Liu, Y. Li, B. Li and Y. J. Chen, Construction of $\mathrm{pH}$ sensitive lysozyme/pectin nanogel for tumor methotrexate delivery, Colloids Surf., B, 2015, 126, 459-466.

9 Y. L. Li, D. Maciel, J. Rodrigues, X. Y. Shi and H. Tomas, Biodegradable polymer nanogels for drug/nucleic acid delivery, Chem. Rev., 2015, 115(16), 8564-8608.

10 T. Farjami and A. Madadlou, Fabrication methods of biopolymeric microgels and microgel-based hydrogels, Food Hydrocolloids, 2017, 62, 262-272.

11 D. Maciel, P. Figueira, S. L. Xiao, D. M. Hu, X. Y. Shi, J. Rodrigues, H. Tomás and Y. L. Li, Redox-responsive alginate nanogels with enhanced anticancer cytotoxicity, Biomacromolecules, 2013, 14(9), 3140-3146.

12 Y. L. Li and C. S. Liu, Nanomaterial-based bone regeneration, Nanoscale, 2017, 9, 4862-4874.

13 Z. Li, W. Xu, Y. Wang, B. R. Shah, C. Zhang, Y. Chen, Y. Li and B. Li, Quantum dots loaded nanogels for low cytotoxicity, pH-sensitive fluorescence, cell imaging and drug delivery, Carbohydr. Polym., 2015, 121, 477-485.

14 W. Li, X. Y. Li, Q. Wang, Y. J. Pan, T. Wang, H. Q. Wang, R. Song and H. B. Deng, Antibacterial activity of nanofibrous mats with lysozyme-layered silicate composites via electro-spraying, Carbohydr. Polym., 2014, 99, 218-225.

15 D. Ren, J. Qi, A. Xie, M. Jia, X. Yang and H. Xiao, Encapsulation in lysozyme/A. Sphaerocephala Krasch polysaccharide nanoparticles increases stability and bioefficacy of curcumin, J. Funct. Foods, 2017, 38, 100-109.

16 X. P. Geng, B. Cui, Y. Li, W. P. Jin, Y. P. An, B. Zhou, T. Ye, L. He, H. S. Liang, L. Wang, Y. J. Chen and B. Li, Preparation and characterization of ovalbumin and carboxymethyl cellulose conjugates via glycosylation, Food Hydrocolloids, 2014, 37, 86-92.

17 Y. B. Song and L. Y. Chen, Effect of net surface charge on physical properties of the cellulose nanoparticles and their efficacy for oral protein delivery, Carbohydr. Polym., 2015, 121, 10-17.

$18 \mathrm{~J}$. Li and X. Wang, Binding of (-)-epigallocatechin-3-gallate with thermally-induced bovine serum albumin/tcarrageenan particles, Food Chem., 2015, 168, 566-571.

19 Y. Song, Y. Zhou and L. Chen, Wood cellulose-based polyelectrolyte complex nano-particles as protein carriers, J. Mater. Chem., 2012, 22(6), 2512-2519.

20 K. K. Zhu, T. Ye, J. J. Liu, Z. Peng, S. S. Xu, J. Q. Lei, H. B. Deng and B. Li, nanogels fabricated by lysozyme and sodium carboxymethyl cellulose for 5-fluorouracil controlled release, Int. J. Pharm., 2013, 441(1), 721-727.

21 H. S. Liang, Q. R. Huang, B. Zhou, L. He, L. F. Lin, Y. P. An, Y. Li, S. L. Liu, Y. J. Chen and B. Li, Self-assembled zeinsodium carboxymethyl cellulose nanoparticles as an effective drug carrier and transporter, J. Mater. Chem. B., 2015, 3(16), 3242-3253.

22 A. P. Rokhade, S. A. Agnibotri, S. A. Patil, N. N. Mallikarjuna, P. V. Kulkarni and T. M. Aminabhavi, Semi-interpenetrating polymer network microspheres of gelatin and sodium carboxymethyl cellulose for controlled release of ketorolac tromethamine, Carbohydr. Polym., 2006, 65, 243-252.

23 I. Baccelli and A. Trumpp, The evolving concept of cancer and metastasis stem cells, J. Cell Biol., 2012, 198, 281-293.

24 M. Ahmad, W. N. Baba, U. Shah, A. Gain and F. A. Masoodi, Nutraceutical properties of the green tea polyphenols, J. Food Process. Technol., 2014, 5, 390.

25 J. H. Cao, J. Han, H. Xiao, J. P. Qiao and M. Han, Effect of tea polyphenol compounds on anticancer drug in terms of antitumor activity, toxicology, and pharmacokinetics, Nutrients, 2016, 3, 762.

26 P. Chanphai, P. Bourassa, C. D. Kanakis, P. A. Tarantilis, M. G. Polissiou and H. A. Tajmir-Riahi, Review on the loading efficacy of dietary tea polyphenols with milk proteins, Food Hydrocolloids, 2018, 77, 322-328.

27 M. G. Sajilata, P. R. Bajaj and R. S. Singhal, Tea polyphenols as nutraceuticals, Compr. Rev. Food Sci. Food Saf., 2008, 7, 229-254.

28 S. Mandel, T. Weinreb Amit and M. B. H. Youdim, Cell signaling pathways in the neuroprotective actions of green tea polyphenol(-)-epigallocatechin-3-gallate: Implications for neurodegenerative diseases, J. Neurochem., 2004, 88, 1555-1569.

29 J. Liang, H. Yan, P. Puligundla, X. L. Gao, Y. B. Zhou and X. C. Wan, Applications of chitosan nanoparticles to enhance absorption and bioavailability of tea polyphenols: A review, Food Hydrocolloids, 2017, 69, 286-292.

30 K. Shanmugaraj, S. Anandakumar and M. Ilanchelian, Probing the binding interaction of thionine with lysozyme: A spectroscopic and molecular docking investigation, Dyes Pigm., 2015, 112, 210-219.

31 A. Shpigelman, Y. Cohen and Y. D. Livney, Thermallyinduced blactoglobulin-EGCG nanovehicles: loading, 
stability, sensory and digestive release study, Food Hydrocolloids, 2012, 29, 57-67.

32 Q. V. Vuong, C. E. Stathopoulos, J. B. Golding, M. H. Nguyen and P. D. Roach, Isolation of green tea catechins and their utilisation in the food industry, Food Rev. Int., 2011, 27, 227-247.

33 Y. G. Guan, J. Wu and Q. X. Zhong, Eugenol improves physical and chemical stabilities of nanoemulsions loaded with $\beta$-carotene, Food Chem., 2016, 194, 787-796.

34 X. L. Huang, Y. Q. Dai, J. X. Cai, N. J. Zhong, H. Xiao, D. J. McClements and K. Hu, Resveratrol encapsulation in core-shell biopolymer nanoparticles: Impact on antioxidant and anticancer activities, Food Hydrocolloids, 2017, 64, 157-165.

35 T. Mosmann, Rapid colorimetric assay to cellular growth and survival: application to proliferation and cytotoxicity assays, J. Immunol. Methods, 1983, 65(1-2), 55-63.

36 I. Vermes, C. Haanen, H. Steffens-Nakken and C. Reutellingsperger, A novel assay for apoptosis flow cytometric detection of phosphatidylserine expression on early apoptotic cells using fluorescein labeled annexin $\mathrm{V}, \mathrm{J}$. Immunol. Methods, 1995, 184(1), 39-42.

37 O. G. Jones and D. J. McClements, Stability of biopolymer particles formed by heat treatment of $\beta$-lactoglobulin/beet pectin electrostatic complexes, Food Biophys., 2008, 3(2), 191-197.

38 J. M. Jung, G. Savin and M. Pouzot, Structure of heat-induced $\beta$-lactoglobulin aggregates and their complexes with sodium-dodecyl sulfate, Biomacromolecules, 2008, 9(9), 2477-2486.
39 M. A. Theresa and R. C. Pieter, Drug delivery systems: Entering the mainstream, Science, 2004, 303(5665), 18181832.

40 C. Schmitt and S. L. Turgeon, Protein/polysaccharide complexes and coacervates in food systems, Adv. Colloid Interface Sci., 2011, 167(1), 333-339.

41 C. J. Souza and E. E. Garcia, Effect of salt and protein concentrations on the association and dissociation of ovalbumin-pectin complexes, Food Hydrocolloids, 2015, 47, 124-129.

42 I. J. Joye, G. Davidov-Pardo and D. J. McClements, Nanotechnology for increased micronutrient bioavailability, Trends Food Sci. Technol., 2014, 40, 168-182.

43 J. Yi, T. I. Lam, W. Yokoyama, L. W. Cheng and F. Zhong, Beta-carotene encapsulated in food protein nanoparticles reduces peroxyl radical oxidation in Caco-2 cells, Food Hydrocolloids, 2015, 43, 31-40.

44 S. Raghavan and H. G. Kristinsson, Antioxidative efficacy of alkali-treated tilapia protein hydrolysates: A comparative study of five enzymes, J. Agric. Food Chem., 2008, 56(4), 1434-1441.

45 A. Tapal and P. K. Tiku, Complexation of curcumin with soy protein isolate and its implications on solubility and stability of curcumin, Food Chem., 2012, 130(4), 960-965.

46 M. G. Ferruzzi and R. J. Green, Analysis of catechins from milk-tea beverages by enzyme-assisted extraction followed by high-performance liquid chromatography, Food Chem., 2006, 99, 484-491.

47 C. Duan, J. Gao, D. Zhang, L. Jia, Y. Liu, D. Zheng and Q. Zhang, Galactose-decorated pH-responsive nanogels for hepatoma-targeted delivery of oridonin, Biomacromolecules, 2011, 12, 4335-4343. 